\title{
Anticoagulation, catheterization, and minimizing intervention: how cutting-edge cuts down disease
}

Welcome to this special issue of Annals of Translational Medicine on the "Anticoagulation, catheterization, and minimizing intervention: how cutting-edge cuts down disease” (edited by Prof. Rami N. Khouzam).

Cardiovascular disease, listed as the underlying cause of death, accounts for over 840,000 deaths in the United States (U.S.). This translates into one out of every three deaths in the U.S. About 2,200 Americans die of cardiovascular disease each day, an average of one death every 40 seconds (1). Innovation is on the rise to prevent cardiovascular death by both man and machine. Efforts are being made in drug development to avoid new "cutting-edge" antithrombotics from morphing into the "bleeding-edge" by making drug therapy safer. We are witnessing the injection of "big" technology in the healthcare market with applications in the form of watches and wearables at the patient level and with new technology available to physicians to monitor patients without invasive means. Finally, we focus on the opportune time to intervene in patients on both ends of the spectrum of critical illness, those clinging to life and those embracing rehabilitation living with cardiovascular disease and taking ownership in their cardiovascular health. Multiple manuscripts in this special issue address and review recent developments in the realm of antithrombotic therapy and reversal, intervention by both invasive and non-invasive means, and current challenges.

\section{Introduction: poem}

As the authors believe in the strong relation between art and science, a nice poem entitled: "Cardiac Technology", by Dr. Pooja S. Jagadish, was used as an introduction to this special issue.

\section{Section 1: antiplatelets and anticoagulation: updated literature}

"The death of aspirin for primary prevention—should aspirin be changed to a prescription only medication?" By Dr. Aranyak Rawal et al. The aim of this editorial is to describe the rise and fall of aspirin as an antiplatelet strategy. This article elaborates on recent studies evaluating the risks and benefits of aspirin for primary prevention of cardiovascular events.

"The dilemma of aspirin resistance in obese patients". By Devarshi Ardeshna et al.

This manuscript addresses the epidemic of obesity that not only increases cardiovascular risk but also decreases aspirin efficacy based on pharmacokinetic and pharmacodynamic studies. Ultimately, a one-dose-fits-all aspirin regimen may not be optimal in obese patients and instead requires tailored therapy.

"From WOEST to AUGUSTUS: a review of safety and efficacy of triple versus dual antithrombotic regimens in patients with atrial fibrillation requiring percutaneous coronary intervention for acute coronary syndrome”. By Dr. David W. Jones et al.

There exist many therapeutic options to prevent thromboembolic events in patients with atrial fibrillation (AF) who undergo PCI with stenting, however there is often hesitation to drop aspirin in the highest risk patients. This manuscript reviews the addressing risks and benefits of the antithrombotic regimens in patients with acute coronary syndrome and concomitant AF.

"Ticagrelor or prasugrel versus clopidogrel in combination with anticoagulation for treatment of acute coronary syndrome in patients with atrial fibrillation”. By Dr. Irene Kirolos, et al.

Clopidogrel is the default P2Y12 inhibitor recommended in patients with acute coronary syndrome and concomitant $\mathrm{AF}$, despite the fact that ticagrelor and prasugrel exhibit a stronger antiplatelet effect. With the robust safety benefit of the novel anticoagulants, perhaps the most intriguing combination may be with the ticagrelor or prasugrel to offset the increased bleeding risk in combination. However, well-designed studies may better inform choice of P2Y12 inhibitor used until this strategy can be widely implemented.

"Post-operative atrial fibrillation: should we anticoagulate?" By Dr. Pooja S. Jagadish, et al. 
Despite low-level evidence in this population, the general consensus per the AHA/ACC/HRS guidelines appears to be that anticoagulation is recommended for patients post-operatively with prolonged duration post-operative AF ( $>48$ hours) with either multiple stroke risk factors or other AF comorbidities.

"Intravenous cangrelor as a peri-procedural bridge with applied uses in ischemic events". By Dr. Venugopal B. Bhattad, et al.

The perioperative management of patients on antiplatelet therapy for cardiovascular and neurological indications who require urgent surgery remains challenging. It is critical to prevent ischemia and thrombosis, while minimizing the risk of bleeding. Successful bridging therapy with cangrelor is promising due to its distinctive pharmacokinetic profile with fast onset and reversibility.

"P2Y12 Inhibitors: do they increase cancer risk?" By Joseph J. Fierro, PharmD, et al.

Despite the alarm of initial findings regarding prolonged treatment with P2Y12 inhibitors and increased incidence of cancer diagnosis, it is unlikely that a significant association exists between cancer dual antiplatelet activity.

"Targeting ticagrelor: a novel therapy for emergency reversal". By Brandon Cave, PharmD, et al.

Despite the routine use of potent P2Y12 inhibitors and investment into reversal therapies for novel anticoagulants, there is a lack of therapeutic options to effectively reverse antiplatelet therapy. The addition of a reversal agent for ticagrelor will increase the safety of patients requiring reversal for acute uncontrolled or life-threatening major bleeding events. PB2452 represents an advancement in this area and is the first reversal agent of its kind in the case of urgent surgical procedures or uncontrolled major or life-threatening bleeding.

"Current status of oral anticoagulant reversal strategies: a review". By Dr. Aranyak Rawal, et al.

Since the emergence of DOACs and the significant increase in their use, there has become a greater need for management of bleeding attributed to their use. Controversies exist on whether specific reversal strategies or non-specific reversal agents should be preferred to reverse DOACs. In this review, the evidence for DOAC reversal is discussed.

\section{Section 2: cardiac catheterization and rehab: when is intervention appropriate?}

"Is early invasive management as ST elevation myocardial infarction warranted in De Winter's sign? A speak into the widowmaker”. By Dr. Joel M. Raja, et al.

Current guidelines advocate immediate cardiac catheterization in cases of ST-segment elevation and presumed new left bundle-branch block on EKG. However, ample evidence also exists for patients presenting with angina, ST-segment depression and peaked $\mathrm{T}$ waves, combined even in the absence of classic ST-segment elevation in the precordial leads of the 12-lead EKG, having significant LAD occlusion. De Winter's sign is often missed by the automated computerized EKG interpretations as well as physicians in the search for the classical ST-segment elevation. This calls for need for educating the physicians interpreting EKGs about De Winter's sign to facilitate early detection and revascularization.

"After COACT trial—new perspectives for the management of NSTEMI. Early vs. late cardiac catheterization post cardiac arrest”. By Dr. Miguel A. Maturana, et al.

For patients with return of spontaneous circulation following an out-of-hospital cardiac arrest and non-ST-segment elevation, the strategy for intervention is unclear. Physicians have struggled with this gray area of medicine, questioning whether to proceed with urgent or delayed coronary angiography. The COACT trial has added new energy to the discussion in the findings that immediate angiography was not superior to delayed angiography for survival at 90 days. This and other studies on the horizon have the potential to solidify the strategy for these scenarios.

"Barriers physicians face when referring patients to cardiac rehabilitation: a narrative review". By Carol Elsakr, et al.

While more study is needed on the awareness physicians have of cardiac rehabilitation (CR), this review overall supports using educational interventions for improving CR referral rates. Furthermore, this review supports targeting various aspects of the referral process itself for improving CR referral rates.

Also, to follow,

"Action plan for improving cardiac rehabilitation-related outcomes in a university hospital based on a review of previous interventions". By Carol Elsakr, et al.

In this manuscript, the authors shared their experience at Methodist University Hospital (MUH) in Memphis, TN, USA; elaborating on an action plan, in order to optimally improve the quality of care for cardiac patients at MUH, the DMAIC 
project set out to improve CR-referral rates with resounding success.

"Cardiac physiology in post myocardial infarction patients: the effect of cardiac rehabilitation programs (CRP)—a systematic review and update meta-analysis". By Dr. Irene Kirolos, et al.

CRP objectively improves various aspects of functional cardiac capacity in post-myocardial infarction patients. This is mediated through higher ejection fraction and peak $\mathrm{VO}_{2}$, lower resting heart rate, left ventricular end diastolic volume and wall motion score index. This effect may explain the reported improvement of functionality and mortality among those patients. Further randomized trials may help evaluate the long-term benefits of CRP.

\section{Section 3: non-invasive monitoring and minimally-invasive procedures: the role of technology in patient safety}

“Apple Watch, wearables, and heart rhythm: where do we stand?" By Dr. Joel Mukesh Raja, et al.

The emergence of consumer use of wearables and smart watches as health monitoring devices as a non-invasive, ambulatory assessment of heart rate and rhythm, is definitely novel. The different wearable technology and its efficacy in detecting $\mathrm{AF}$ is well demonstrated in the literature. However, false positives can cause undue anxiety to the user and may drive up health costs with unwarranted emergency visits. Irregular rhythm notifications in people without a known diagnosis of AF supported by physician review can help in uncovering the subclinical AF population. Wearable technology may not serve as the ultimate tool for diagnosis of AF, rather a nidus for the general population to seek medical advice on being notified of having an irregular rhythm for confirmation potentially leading to better outcomes.

"Wearable cardioverter defibrillators (WCD): dead on arrival or chance of survival?” By Dr. Pooja S. Jagadish, et al.

As it stands, the WCD has a class IIb recommendation for primary prevention of arrhythmias in post-myocardial infarction patients with ischemic heart disease and a left ventricular ejection fraction (LVEF) below 40\%. Additionally, WCD are recommended for secondary prevention in newly diagnosed non-ischemic cardiomyopathy patients with class II-III heart failure and an LVEF below 35\%. There are device modifications that can improve function, device use, and perhaps even expand its market and functionality, warranting reconsideration of its validity.

"CardioMEMS: where we are and where can we go?" By Dr. Issa Pour-Ghaz, et al.

Due to the ever-growing costs associated with health care admissions, finding solutions for monitoring of chronic conditions is necessary. In recent years there have been a series of new devices created to address this role. Hemodynamic monitoring with CardioMEMS has shown to be effective in prevention and reduction of HF hospitalizations in patients with HFrEF and HFpEF. CardioMEMS is a great option for pulmonary hypertension monitoring to avoid the need for repeated cardiac catheterization. This application can also lead to easier medication management in these patients and worth further investigation. With future iterations of this device, further research may lead to a great impact in the future.

"Is there an optimal "door to cath time" in the treatment of acute pulmonary embolism with catheter-directed thrombolysis?” By Dr. Aranyak Rawal, et al.

While catheter directed thrombolysis is an effective management strategy for acute pulmonary embolism, there is no consensus available for the optimal timing of initiation of therapy. Based on review of the available literature, ultrasound assisted catheter directed thrombolysis (UACT) may provide benefit for patients started on therapy early $(<24-48$ after presentation) compared to those in whom UACT is delayed. However, further studies are needed to further clarify the role, benefit and best timing of UACT.

"Transcarotid transcatheter aortic valve replacement (TAVR) with a focus on transcarotid: a review of the current literature". By Dr. Issa Pour-Ghaz, et al.

TAVR is rapidly becoming the standard of care in patients with moderate to severe aortic valve stenosis. The most common site for access is the femoral route, although a multitude of other sites are available. Transfemoral TAVR is problematic in patients with poor peripheral access and vascular disease; hence, alternative routes of access are crucial. Among the other access sites, the transcarotid approach has proven to be a promising alternative with comparable results and low complications.

"Accuracy of noninvasive and minimally invasive hemodynamic monitoring: where do we stand?" By Dr. Issa Pour-Ghaz, et al.

When it comes to blood pressure and hemodynamic monitoring, invasive arterial cannulation and pulmonary artery 
catheter are the gold standards in acute and critical care settings. Noninvasive methods are a safe alternative for hemodynamic monitoring in patients who are in critical or non-critical states. These devices have less complications, are less expensive and can be used in patients who need more in-depth monitoring of their hemodynamic status.

"The renal resistive index as a new complementary tool to predict microvascular diabetic complications in children and adolescents: a groundbreaking finding”. By Dr. Abeer Ahmed Abdel Maksoud, et al.

Correlations between renal resistive index (RI) and different clinical and biochemical parameters in each patients group were studied in a trial to find out possible contributing factors for increased renal RI in type 1 diabetes mellitus. Results showed that RI is correlated positively at a duration above 10 years, HbA1c above $7.5 \mathrm{mg} / \mathrm{dL}$, and serum triglycerides. Meanwhile, renal RI was not significantly correlated to age, BMI, serum lipids or other studied parameters. The current study may suggest the use of renal RI as a useful complementary test for the evaluation of functional alterations in renal hemodynamics in early stages of diabetic nephropathy and possible concurrent microvascular diabetic complications.

In conclusion, the authors are reviewing both most interesting and cutting-edge up-to-date cardiovascular topics. There is a special emphasis on the rate of technological advances and patient safety, specifically in regard to antithrombotic selection and targeted reversal, and which should take priority in various scenarios. Additionally, limiting patient exposure to adverse events through non-invasive monitoring can be extremely useful and allow selection of only those with the highest risk for further invasive treatment. Finally, it is likely only the beginning of the technology boom that will aim to cut down disease. For instance, artificial intelligence is already being tested for its usefulness in medicine. However, one of the greatest technological minds of our generation, Steve Jobs, once said, "Technology is notbing. What's important is that you bave a faith in people, that they're basically good and smart, and if you give them tools, they'll do wonderful things with them." We, as physicians, will be provided with extraordinary tools throughout our career that will allow us to do wonderful things, but also we must show faith in our patients as they also acquire these tools, to encourage them to do wonderful things with their own health as well.

Once again, we wish to thank the authors for their original and valuable contribution, hoping that this volume may be of substantial interest for the readership of the journal.

\section{Acknowledgments}

I would like to acknowledge Brandon Cave, PharmD, and Poojitha Jagadish, MD for their great contributions with the conception and design of multiple topics of this issue as well as writing and helping in editing of manuscripts.

\section{Footnote}

Conflicts of Interest: The author has no conflicts of interest to declare.

Ethical Statement: The author is accountable for all aspects of the work in ensuring that questions related to the accuracy or integrity of any part of the work are appropriately investigated and resolved.

\section{References}

1. Khouzam RN. Advances in Cardiology, Interventional Cardiology, Electrophysiology, and Structural Heart Disease: A Moving Target. Ann Transl Med 2018;6:1. 


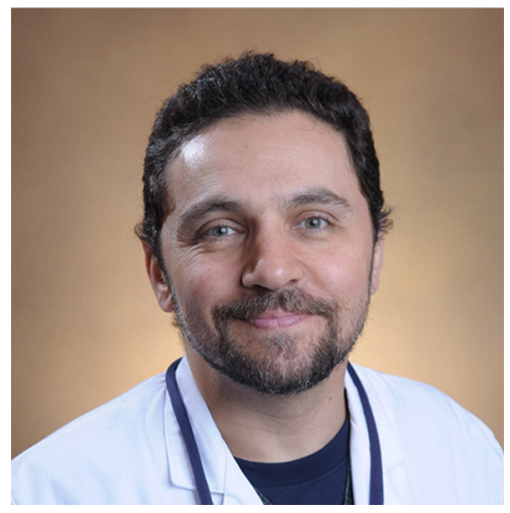

Rami N. Khouzam

\section{Rami N. Khouzam, MD, FACC, FACP, FASNC, FASE, FSCAI}

Department of Medicine, Division of Cardiovascular Diseases,

University of Tennessee Health Science Center, Cardiovascular Institute,

Methodist Le Bonheur Healthcare System, Memphis, TN, USA.

(Email: khouzamrami@yahoo.com) doi: $10.21037 / \mathrm{atm} .2019 .07 .75$

View this article at: http://dx.doi.org/10.21037/atm.2019.07.75

Cite this article as: Khouzam RN. Anticoagulation, catheterization, and minimizing intervention: how cutting-edge cuts down disease. Ann Transl Med 2019;7(17):401. doi: 10.21037/ atm.2019.07.75 